\title{
Free-Surface Modelling Technology for Compressible and Violent Flows
}

\author{
Johan A. Heyns* \\ Council for Scientific and Industrial Research, Pretoria, Gauteng, South Africa \\ Thomas M. Harms ${ }^{\dagger}$ \\ University of Stellenbosch, Stellenbosch, Western Cape, South Africa \\ Arnaud G. Malan ${ }^{\ddagger}$ \\ University of Cape Town, Cape Town, Western Cape, South Africa
}

\begin{abstract}
This study presents the development of novel modelling technology for compressible and violent free-surface flows, where the new technology aims to extend the capabilities of existing FSM formulations. For the purpose of this study the volume-of-fluid (VOF) method is extended in two ways: Firstly, we aim to improve on the accuracy of existing free-surface interface capturing schemes, and secondly, a newly developed weakly compressible formulation is introduced. The proposed interface capturing formulation reduces the degree of numerical smearing, while maintaining the integrity of the interface shape. It involves combining the approaches of blended higher-resolution discretisation and adding an artificial compressive term in a manner which retains the strength of each. The weakly compressible formulation proposes an altered governing equation set which accurately accounts for large variance in gas density at low Mach numbers and may be solved at little additional computational cost. All governing equations are discretized via an unstructured edge-based vertex centred finite volume method, and solved via a parallel implicit solver. The newly developed technology is validated through application to various benchmark test cases.
\end{abstract}

\section{Nomenclature}

$\mathcal{A} \quad$ Outward pointing area vector, $\mathrm{m}^{2}$

a Acceleration, $\mathrm{m} / \mathrm{s}^{2}$

$E_{\text {comp }}$ Comparative error

$E_{\text {diff }}$ Diffusive error

d Vector between donor and acceptor cell, $\mathrm{m}$

C Edge coefficient, $\mathrm{m}^{2}$

c Acoustic velocity, $\mathrm{m} / \mathrm{s}$

$c_{f} \quad$ Courant number

$g \quad$ Gravitational acceleration, $\mathrm{m} / \mathrm{s}^{2}$

$\mathcal{K} \quad$ Arbitrary coefficient

n Unit vector

$p \quad$ Pressure, $\mathrm{N} / \mathrm{m}^{2}$

$r^{*} \quad$ Normalised slope gradient

$t \quad$ Time, $\mathrm{s}$

$u_{i} \quad$ Velocity component in cartesian coordinate direction $i, \mathrm{~m} / \mathrm{s}^{2}$

$S \quad$ Source term

*Student, Aerospace Systems Competency, jheyns@csir.co.za, SS.

${ }^{\dagger}$ Mechanical Engineering, tmh@sun.ac.za.

${ }_{\ddagger}$ Mechanical Engineering, arnaud.malan@uct.ac.za. 


$\begin{array}{ll}\mathcal{V} & \text { Volume, } \mathrm{m}^{3} \\ x_{i} & \text { Cartesian spatial coordinate component } i, \mathrm{~m} \\ \text { Greek } & \text { symbols } \\ \alpha & \text { Volume fraction } \\ \gamma & \text { Weighting factor } \\ \mu & \text { Viscosity, } \mathrm{kg} / \mathrm{m} \mathrm{s} \\ \phi & \text { Arbitrary scalar value } \\ \rho & \text { Density, } \mathrm{kg} / \mathrm{m}^{3} \\ \tau & \text { Pseudo time } \\ \text { Subscript } \\ A & \text { Acceptor cell } \\ D & \text { Donor cell } \\ c & \text { Compressive } \\ f & \text { Face value } \\ g & \text { Gas } \\ H C & \text { Hyper-C } \\ l & \text { Liquid } \\ o & \text { Initial value } \\ U & \text { Upwind cell } \\ U Q & \text { ULTIMATE-QUICKEST }\end{array}$

\section{Introduction}

Various industries benefit from the accurate modelling of immiscible two fluid flow. These include the casting industry, maritime and naval engineering (where impact loads on fixed and floating structures are studied) as well as in the transportation of fuel and other fluids by means of surface or air. As computational hardware and modelling techniques improve, the fidelity of numerical models increases along with the ability to model more complex flow phenomena with greater efficiency. Free-surface modelling (FSM) is commonly used to refer to the modelling of immiscible two-fluid flow by means of computational fluid dynamics (CFD). To date, most free-surface models are used to simulate relatively moderate flows and assume incompressible flow. This study considers the extension of FSM technology for violent and compressible flow of high density ratio two-fluid systems.

Various aspects of FSM are continuously reviewed with the aim of improving the accuracy. Particularly where violent or complex flow dynamics is involved, further development is required in the prediction of the interface evolution. Various approaches for the latter ${ }^{1-4}$ exist, where for this work the conservative volumeof-fluid $(\mathrm{VOF})^{5}$ method is preferred as it is computationally efficient and capable of modelling complex flow phenomena such as separation and merging of the interface. ${ }^{6}$ With the VOF method the fluids are modelled by means of an advecting volume fraction equation and when applied to violent flow conditions it may result in severe numerical smearing of the interface.

With regards to compressibility of the fluids in FSM analysis, most existing models treat both gas and liquid as incompressible, neglecting the effect of changes in density due to pressure. This is a reasonable assumption for many free-surface flows, however in high density ratio systems, subjected to large excitations, entrapped gas pockets may be subjected to notable fluctuations in pressure. This is due to significant changes in the static pressure of the high density liquid. In these cases, the compressibility of the gas can greatly influence the prediction of the calculated pressures.

In light of the above, for the purpose of this paper two areas within FSM is reviewed and extended: Firstly, a new free-surface capturing formulation is presented, which aims to improve on the accuracy of existing VOF surface tracking algorithms. In so doing, providing a scheme that maintains a well defined interface shape, while ensuring interface sharpness is retained. Secondly, a weakly compressible formulation for VOF is presented for low Mach number flows, that takes into account density changes in the gas due to large static pressure variations in the liquid. The set of governing equations presented, therefore accurately accounts for the compressibility of the gas in a manner which is suitable for an efficient numerical solution. The resulting formulations are discretised and solved via a finite volume, edge-based approach, ${ }^{7}$ which has 
been shown to be accurate as well as computationally efficient ${ }^{8}$ and furthermore is naturally applicable to hybrid unstructured meshes. In Section II of this paper the surface capturing methodology for the VOF approach is reviewed and a new formulation is presented and evaluated. Section III details a newly developed weakly compressible formulation for VOF.

\section{I.A. Finite volume, vertex centre, edge-based formulation}

To serve as a backdrop for the work presented here, only a general introduction to the finite volume, vertex centre, edge-based approach is provided. It is asked that the reader refer to the cited literature ${ }^{7}$ for a more detailed account of the spatial discretisation.

With the said finite volume approach, the spatial domain, $\mathcal{V}$, is subdivide into a finite number of nonoverlapping volumes $\mathcal{V}_{\xi} \in \mathcal{V}$ as illustrated in figure 1 . When considering a general advection-diffusion of a arbitrary scalar value, $\phi$, the governing equation reads

$$
\frac{\partial \phi}{\partial t}+\frac{\partial \phi u_{i}}{\partial x_{i}}-\frac{\partial}{\partial x_{i}}\left(\mathcal{K} \frac{\partial \phi}{\partial x_{i}}\right)=0
$$

which can be cast into weak form for the volume $\mathcal{V}_{\xi}$ and by means of the divergence theorem can be written in terms of surface integrals

$$
\int_{\mathcal{V}_{\xi}} \frac{\partial \phi}{\partial t} d \mathcal{V}+\int_{\mathcal{A}_{\xi}} \phi u_{i} \mathbf{n}_{i} d \mathcal{A}-\int_{\mathcal{A}_{\xi}} \mathcal{K} \frac{\partial \phi}{\partial x_{i}} \mathbf{n}_{i} d \mathcal{A}=0
$$

where $\mathcal{A}_{\xi}$ is the surface bounding $\mathcal{V}_{\xi}$ and $\mathbf{n}$ is the unit vector normal to the boundary segment $\mathcal{A}$ pointing outward. Further, bounding surface information is stored in an edge-wise manner, as edge-coefficients, to exploit the computational advantages of an edge-based assembly. The edge-coefficients are defined as

$$
\mathbf{C}_{f}=\mathbf{n}_{f_{1}} \mathcal{A}_{f_{1}}+\mathbf{n}_{f_{2}} \mathcal{A}_{f_{2}}
$$

where $f$ denotes the face value.

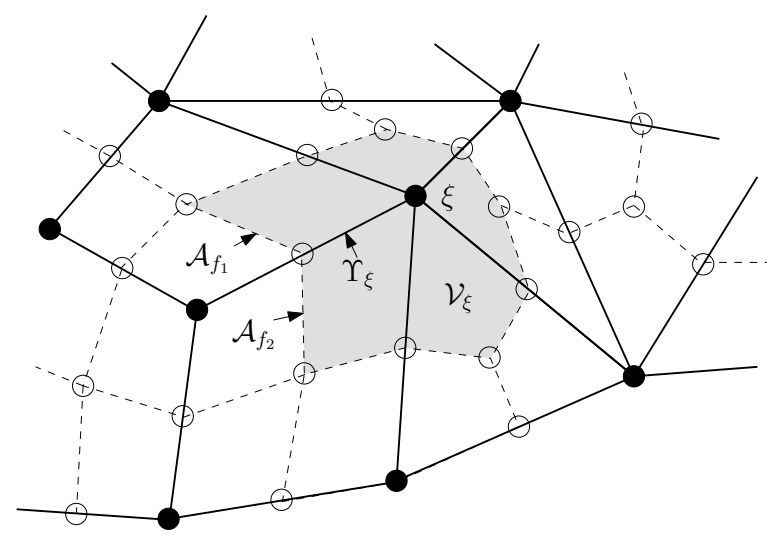

Figure 1: Schematic of the construction of the median-dual-mesh on hybrid grids

\section{Fast compressive surface capturing formulation}

To ensure that the flow characteristics are modelled accurately with FSM, it is necessary to describe the evolution of the free-surface interface accurately. Various methods to describe this evolution of the interface have been described in literature. These include Lagrangian surface fitting methods where the interface is fitted to the mesh; ${ }^{4,9,10}$ Eulerian front tracking techniques or Eulerian volume tracking methods. Front tracking methods include massless particles on the interface, ${ }^{1,11,12}$ height functions ${ }^{13,14}$ and level set methods. ${ }^{2,15,16}$ Whereas with volume tracking methods the fluids are either represented with mass-less particles or with a volume fraction indicator function. 
Volume tracking by means of the advection of a volume fraction is used in this study to describe the evolution of the free-surface interface. This method, commonly referred to as volume-of-fluid approach, is preferred as it conserves mass ${ }^{17-19}$ is capable of modelling complex interface phenomena such as separation as well as merging of the interface; and furthermore is computationally efficient. To ensure a bounded solution while maintaining a sharp interface existing practices advocate either discretizing the volume fraction advactive term via non-linear higher-resolution schemes, or an artificial compressive term is added to the VOF equation.

Variants of the non-linear higher-resolution method include STACS, ${ }^{3}$ CICSAM $^{6}$ and HRIC. ${ }^{20}$ These schemes tend to be computationally efficient, are easily implemented on unstructured three-dimensional meshes and can model complex flow phenomena such as separation and merging of the interface. They are however subjected to numerical smearing, where the degree of smearing is directly proportional to the Courant number. Rusche, ${ }^{21}$ based on a concept of Jasak and Weller, ${ }^{22}$ on the other hand introduced an artificial compressive term in the VOF equation to achieve the necessary compression of the interface. Gopala and Van Wachem ${ }^{18}$ however showed the resulting Inter-Gamma scheme ${ }^{22}$ with the artificial compressive term, is capable of maintaining a sharp interface, but tends to wrinkle the shape of the free-surface. In an effort to improve on the above shortcomings, this paper presents a new surface capturing formulation, which is detailed in the next section.

\section{II.A. Fast compressive surface capturing formulation}

The newly proposed formulation, FCSCF, aims at reducing Courant number related smearing while maintaining a representative interface topology. The method involves discretising the VOF advective term using a blended higher-resolution scheme, but in addition introduces an artificial compressive term to the VOF equation. As a compressive higher-resolution scheme is used in the discretisation of the advective term the degree of compression required from the artificial term is reduced. This prevents the wrinkling of the interface associated with the latter. The resulting formulation written in semi-discrete weak form reads

$$
\frac{\partial \alpha}{\partial t} \int_{\mathcal{V}_{\xi}} \partial \mathcal{V}+\sum_{\Upsilon_{\xi} \cap \mathcal{V}_{\xi}} \alpha_{f} u_{f}^{j} C_{f}^{j}+\sum_{\Upsilon_{\xi} \cap \mathcal{V}_{\xi}} \alpha_{f}\left(1-\alpha_{f}\right) u_{c f}^{j} C_{f}^{j}=0
$$

where $\alpha_{f}$ denotes the volume fraction face value. The compressive velocity is calculated as

$$
u_{c f}=c_{\alpha}\left|u_{f}\right| \mathbf{n}_{\alpha}^{*}
$$

and $u_{f}$ is the velocity face value. For FCSCF it is found that best results are obtained for $c_{\alpha}=0.1$. A smoothed volume fraction value, $\alpha^{*}$, is used to calculate the interface inward pointing normal vector as

$$
\mathbf{n}_{\alpha}^{*}=\frac{\nabla \alpha^{*}}{\left|\nabla \alpha^{*}\right|}
$$

and $\alpha^{*}$ is calculated by solving a diffusive type equation for 2 or 3 pseudo time iterations

$$
\frac{\partial \alpha^{*}}{\partial t_{\tau}}+\frac{\partial}{\partial x_{i}}\left(\frac{\partial \alpha^{*}}{\partial x_{i}}\right)=0
$$

For a sharp, bounded solution which is free from numerical oscillations, the volume fraction face value, $\alpha_{f}$, is discretised using the normalised variable (NV) approach $^{23}$ with convective boundedness criteria (CBC). The normalised variable is defined as

$$
\tilde{\alpha}=\frac{\alpha-\alpha_{U}}{\alpha_{A}-\alpha_{U}}
$$

where the upwind and acceptor cells are denoted respectively $U$ and $A$. For an unstructured formulation, the expression for the projected upwind value as presented by Ubbink and Issa ${ }^{6}$ is used

$$
\alpha_{U}^{*}=\alpha_{A}-2(\nabla \alpha)_{D} \cdot \mathbf{d}
$$

where $D$ denotes the donor cell and the edge vector is defined as $\mathbf{d}=\mathbf{x}_{A}-\mathbf{x}_{D}$. 
By applying CBC, a oscillatory free solution is obtained that remains within the physical bounds of zero and one. For explicit flow calculations CBC reads:

$$
\begin{array}{cl}
\tilde{\alpha}_{D} \leq \tilde{\alpha}_{f} \leq \min \left\{1, \frac{\tilde{\alpha}_{D}}{c_{f}}\right\} & \text { for } 0 \leq \tilde{\alpha}_{D} \leq 1 \\
\tilde{\alpha}_{f}=\tilde{\alpha}_{D} & \text { for } \tilde{\alpha}_{D}<0 \text { or } \tilde{\alpha}_{D}>1
\end{array}
$$

In figure 2 the dark gray area represents the region for which $\mathrm{CBC}$ holds for an explicit solution and the lighter gray area represents the extended region where $\mathrm{CBC}$ holds for an implicit solution. By following the upper bound of $\mathrm{CBC}$ the most compressive solution is obtained.

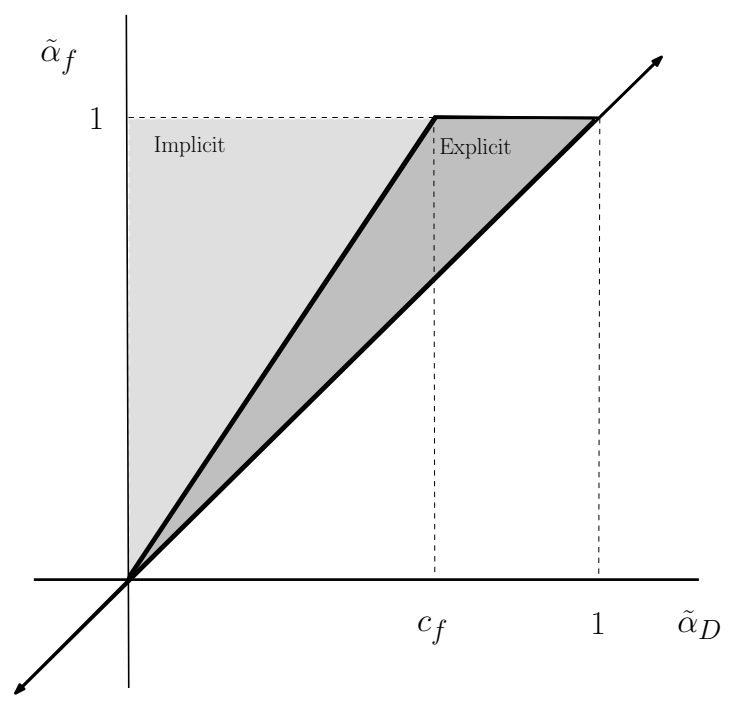

Figure 2: Bounded regions on the normalised variable diagram for explicit and implicit flow calculations

Ubbink $^{24}$ showed that second-order Crank-Nicolson reduces the numerical smearing associated with the temporal discretisation. It is therefore proposed that a Jacobi type dual-time stepping formulation with second order Crank-Nicholson be used for FCSCF.

$$
\begin{aligned}
\frac{\alpha^{\tau+1}-\alpha^{\tau}}{\Delta t_{\tau}}= & -\frac{1}{2}\left[\left.\frac{\partial\left(u_{i} \alpha\right)}{\partial x_{i}}\right|^{\tau}+\left.\frac{\partial\left(u_{i} \alpha\right)}{\partial x_{i}}\right|^{n}\right] \\
& -\left.\frac{\partial}{\partial x_{i}}\left(\left.u_{c}\right|_{i} \alpha(1-\alpha)\right)\right|^{\tau}-\frac{\alpha^{\tau}-\alpha^{n}}{\Delta t}
\end{aligned}
$$

As the solution converges in pseudo time the implicit solution is approached, while the availability criteria is satisfied every time step $\tau$.

The volume fraction face value, $\alpha_{f}$, is discretised using a blended high-resolution scheme that switches between compressive and higher resolution schemes based on the alignment of the free surface interface and the mesh. This ensures that a sharp interface is maintained, while it prevents wrinkling of the interface. The compressive Hyper-C method ${ }^{23}$ is reformulated in the interest of greater numerical efficiency. This is done by expanding the normalised variables as

$$
\alpha_{f_{H C}}= \begin{cases}\min \left\{\alpha_{A}, \frac{\alpha_{D}-\alpha_{U}^{*}}{c_{f}}+\alpha_{U}^{*}\right\} & \text { for } r^{*}>1 \text { and } \alpha_{D}>\alpha_{U}^{*} \\ \max \left\{\alpha_{A}, \frac{\alpha_{D}-\alpha_{U}^{*}}{c_{f}}+\alpha_{U}^{*}\right\} & \text { for } r^{*}>1 \text { and } \alpha_{D}<\alpha_{U}^{*} \\ \alpha_{D} & \text { for } r^{*} \leq 1\end{cases}
$$

where the gradient, $r^{*}$ is defined as

$$
r^{*}=\frac{\alpha_{A}-\alpha_{U}^{*}}{\alpha_{D}-\alpha_{U}^{*}}
$$


In a similar manner the normalised variables of the more diffusive ULTIMATE-QUICKEST ${ }^{23}$ is expanded. For FCSCF the following formulation is used

$$
\tilde{\alpha}_{f_{U Q}}= \begin{cases}\min \left\{k^{*}, \alpha_{f_{H C}}\right\} & \text { for } r^{*}>1 \text { and } \alpha_{D}>\alpha_{U} \\ \max \left\{k^{*}, \alpha_{f_{H C}}\right\} & \text { for } r^{*}>1 \text { and } \alpha_{D}<\alpha_{U} \\ \alpha_{D} & \text { for } r^{*} \leq 1\end{cases}
$$

where $r^{*}$ is the same as in Eq. (13) and

$$
k^{*}=\alpha_{U}+\left[\frac{3+c_{f}}{4}\right]\left(\alpha_{D}-\alpha_{U}\right)+\frac{3\left(1-c_{f}\right)}{8}\left(\alpha_{A}-\alpha_{U}\right)
$$

A weighting factor, $\gamma$, is used to switch between Hyper-C and ULTIMATE-QUICKEST based on the alignment of the free-surface interface and the mesh

$$
\tilde{\alpha}=\gamma_{f} \tilde{\alpha}_{f_{H C}}+\left(1-\gamma_{f}\right) \tilde{\alpha}_{f_{U Q}}
$$

where it is proposed that the following computationally efficient formulation be used to calculate the weighting function

$$
\gamma_{f}=\min \left(\left(\eta_{f}\right)^{m}, 1\right)
$$

and

$$
\eta_{f}=\left|\frac{(\nabla \alpha)_{D} \cdot d_{f}}{\left|(\nabla \alpha)_{D}\right| \cdot\left|d_{f}\right|}\right|
$$

Via numerical experimentation it is found that the best results are obtained if a value of $m=2$ is used and the new formulation reduces the computational cost by up to $60 \%$ when compared to CICSAM. By application to a number of bench-marked test cases FCSCF is compared to a state-of-the-art higher-resolution scheme.

\section{II.B. Evaluation of FCSCF}

FCSCF is evaluated by applying it to a number of benchmark problems presented in literature. To asses the increased accuracy, the predicted results are compared to CICSAM. The reason for the latter is two fold: Firstly, FCSCF builds on CICSAM, and seond, CICSAM is typically employed in benchmarking the stateof-the-art schemes. ${ }^{18,25,26}$ The criteria most commonly used to quantify the accuracy of a VOF surface capturing scheme is the so called comparative error. ${ }^{6,18,27}$ Where an analytical solution is available this reads

$$
E_{\text {comp }}=\frac{1}{N} \sum_{i=1}^{N}\left|\alpha_{\text {analt }}-\alpha_{i}\right|
$$

which provides an indication of the degree of interface deformation and smearing. For test cases where the analytical solution is unknown, it is proposed that the following error formulation be used to evaluate the associated numerical smearing of the interface

$$
E_{\text {diff }}=\frac{4}{N} \sum_{i=1}^{N}\left|\alpha_{i}\right|\left|1-\alpha_{i}\right|
$$

where $E_{d i f f}$ is equal to zero if there are no partially filled cells present.

The rotating key test case (see figure 3 ) is used by various authors ${ }^{3,18,24,28,29}$ to evaluate different surface capturing schemes. A unidirectional velocity field is applied where $u=-\pi / 2\left(y-y_{0}\right)$ and $v=\pi / 2\left(x-x_{0}\right)$ where the rotating key centre is $\left(x_{0}, y_{0}\right)=(25,25) \mathrm{mm}$. A 70 by 70 structured mesh as shown, is used for this analysis. 


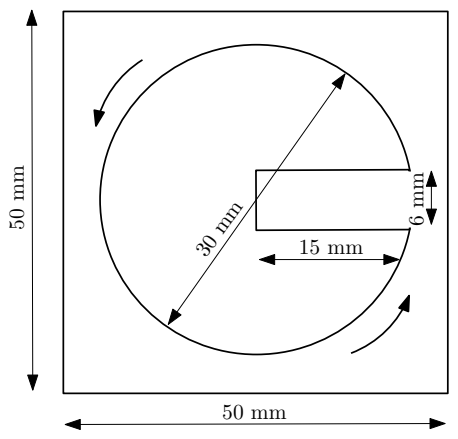

(a) Rotating Key

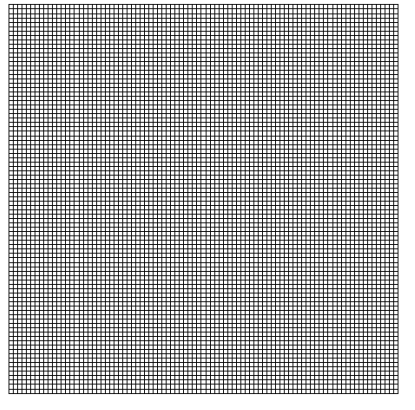

(b) 70x70 structured mesh

Figure 3: Schematic representation of the rotating key together with the computational mesh used

After one rotation the numerical results are evaluated and compared to the analytical solution. In figure 4 the comparative error is plotted as a function of the Courant number. It is found that FCSCF offers considerable improvements in accuracy at higher Courant numbers. Contour plots of the solution after one rotation for both CICSAM and FCSCF are shown in figure 5. From the contour plots it is also clear that FCSCF captures a sharper interface and accurately maintains the shape of the interface.

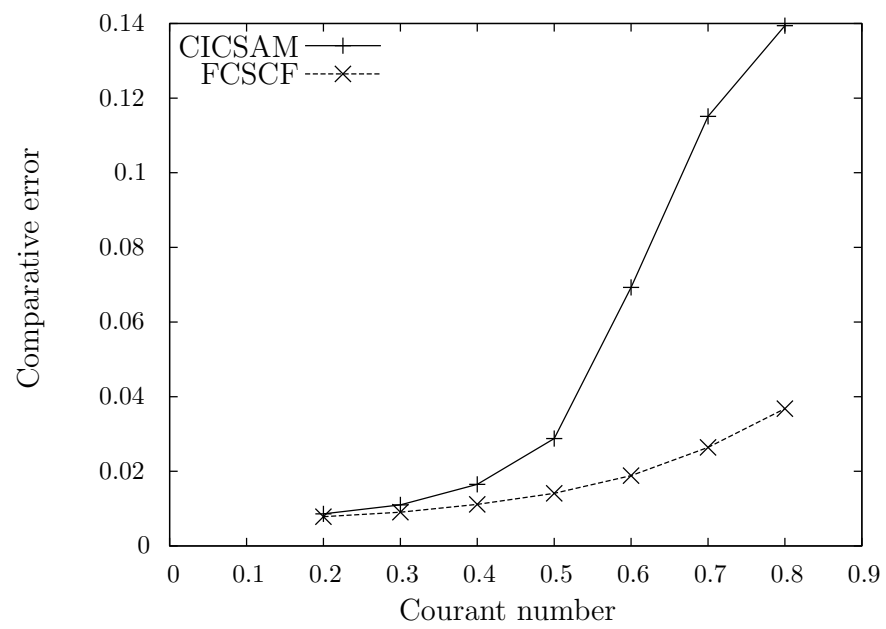

Figure 4: Comparative error as a function of the Courant number for the rotating key

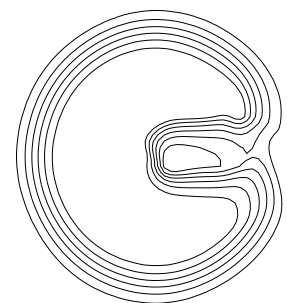

(a) CICSAM

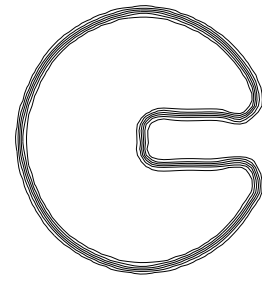

(b) FCSCF

Figure 5: Contour plots of the rotating key for $c_{f}=0.6$

To evaluate the performance characteristics of FCSCF when coupled to a incompressible fluid solver, a falling droplet is modelled. A $50 \mathrm{~mm}$ diameter droplet is placed at the centre of a $100 \mathrm{~mm}$ by $100 \mathrm{~mm}$ computational domain and subjected to a gravitational acceleration of $9.81 \mathrm{~m} / \mathrm{s}^{2}$. For the purpose of this 
analysis no surface tension model is employed, as the aim is to evaluate the numerical smearing inherent to the surface capturing schemes. A Courant number of 0.2 is employed for this analysis.

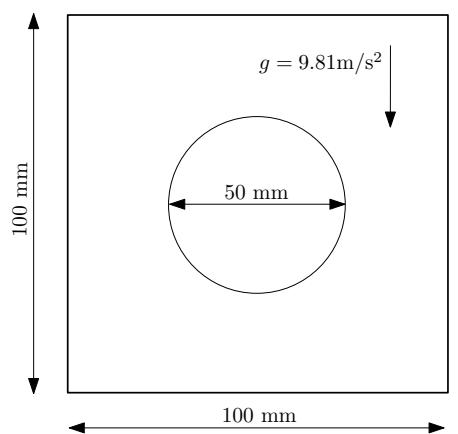

(a) Falling droplet

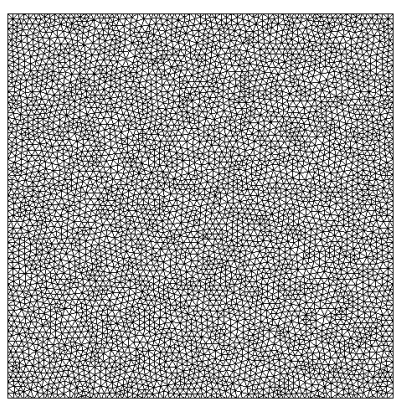

(b) Unstructured mesh

Figure 6: Schematic representation of the falling droplet and the unstructured mesh with 5000 nodes used for the analysis

The diffusive error for FCSCF and CICSAM are evaluated and compared in figure 7. Snapshots of the droplet for the two different schemes at different time steps are shown in figure 8. In the snapshots the high density liquid is coloured blue and the low density gas is taken to be green, partially filled cells are shades of white depending on the degree to which the cell is filled.

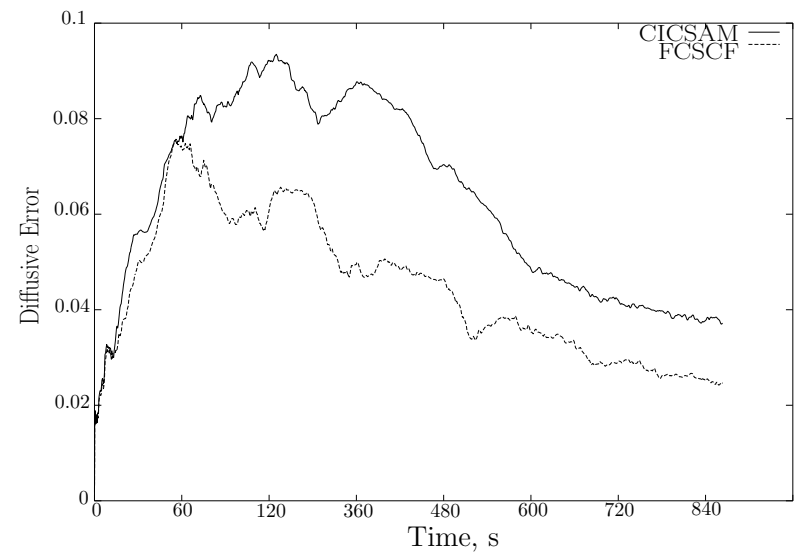

Figure 7: Diffusive error as a function of time for the falling droplet 


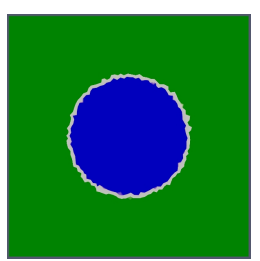

(a) CICSAM, $0 \mathrm{sec}$

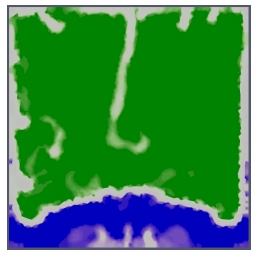

(e) CICSAM, 1 $\min$

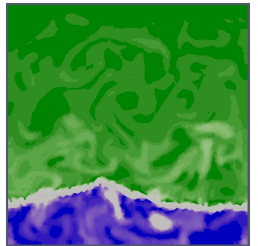

(i) CICSAM, $4 \mathrm{~min}$

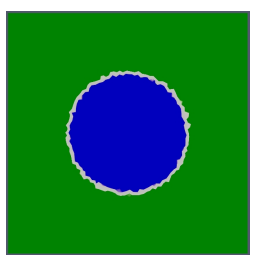

(b) FCSCF, 0 sec

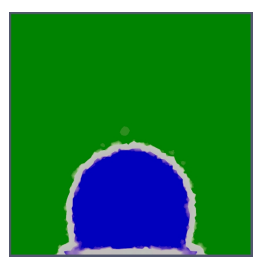

(c) sec

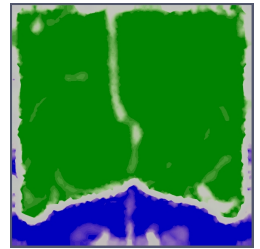

(f) FCSCF, 1 min

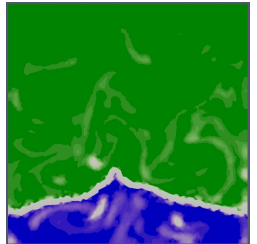

(j) FCSCF, 4 min

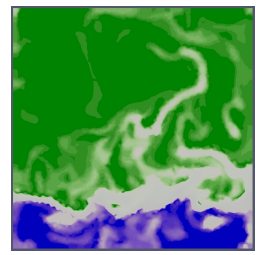

(g)

$\min$

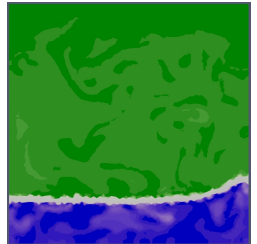

(k) CICSAM, 8 $\min$

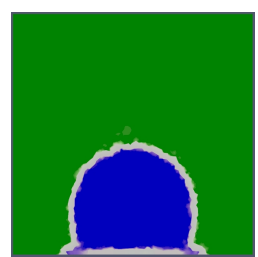

(d) FCSCF, $15 \mathrm{sec}$

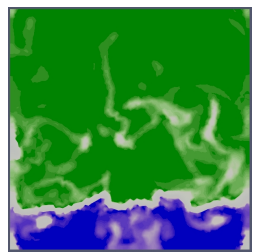

(h) FCSCF, 2 min

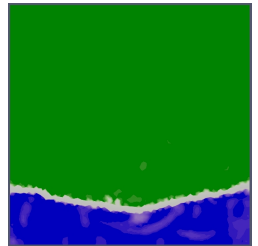

(l) $\mathrm{FCSCF}, 8 \mathrm{~min}$

Figure 8: Snapshots of the falling droplet for CICSAM and FCSCF

From both the error plot as well as the snapshots it is noted that initially the numerical diffusion is similar for the two schemes, but after 60 seconds when the flow becomes more violent it is found that FCSCF achieves up to $40 \%$ less smearing. Further, FCSCF tends to recover interface sharpness whereas the other method tends rather to preserve the smeared interface.

\section{Weakly compressible formulation for the volume-of-fluid free-surface modelling approach}

Godderidge et al. ${ }^{30}$ and Wemmenhove et al. ${ }^{31}$ notes that under certain free-surface flow conditions localised regions within the gas are subjected to large variations in pressures, which results in a change in the fluid characteristics of the system. Experiments conducted by Faltinsen et al. ${ }^{32}$ Lugni et al. ${ }^{33}$ as well as Bullock et al. ${ }^{34}$ showed that the compressibility of air has a significant effect on the impact pressures measured on the tank walls. They noted that presence of the compressible air reduces the peak pressure levels, but increases the duration of the impact.

Various authors ${ }^{35-37}$ have presented high fidelity compressible multi-fluid models. These typically employ the seven equation Baer-Nunziato type model, where two-fluid flow is described by two continuity equations, two momentum equations, two energy equations and a topological or interface equation. With high density ratio systems as are considered in this study, the material properties might vary by three orders of magnitude, rendering the problem numerically stiff. Furthermore, Mach numbers of the flow under consideration are exceedingly low, resulting in the restricted real time step being very small. Using this high fidelity approach to model highly dynamic systems over an extended amount of time is therefore computationally overly expensive.

In line with the above a new weakly compressible formulation for the volume-of-fluid free-surface modelling approach is presented, where after it is evaluated by considering various bench-marked test cases. 


\section{III.A. Proposed formulation}

As noted above, Romenski and Toro ${ }^{37}$ presented a seven equation model that describe the two-fluid flow. For immiscible two-fluid flow, a homogenous flow model with an averaged cell velocity can be assumed, as the time scale on which the turbulent drag forces tend to equalise the velocity is much smaller than the time scales on which the flow is averaged. ${ }^{38}$ Furthermore, the pressures of the liquid and the gas are assumed to be in equilibrium and as a weakly compressible formulation is employed iso-thermal flow can be assumed. From these assumptions the seven equation model reduces to the continuity and momentum equations of respectively the liquid and gas.

$$
\begin{array}{r}
\frac{\partial\left(\alpha_{l} \rho_{l}\right)}{\partial t}+\frac{\partial\left(\alpha_{l} \rho_{l} u_{j}\right)}{\partial x_{j}}=0 \\
\frac{\partial\left(\alpha_{l} \rho_{l} u_{i}\right)}{\partial t}+\frac{\partial\left(\alpha_{l} \rho_{l} u_{i} u_{j}\right)}{\partial x_{j}}+\alpha_{l} \frac{\partial p}{\partial x_{i}}=\alpha S_{l i} \\
\frac{\partial\left(\alpha_{g} \rho_{g}\right)}{\partial t}+\frac{\partial\left(\alpha_{g} \rho_{g} u_{j}\right)}{\partial x_{j}}=0 \\
\frac{\partial\left(\alpha_{g} \rho_{g} u_{i}\right)}{\partial t}+\frac{\partial\left(\alpha_{g} \rho_{g} u_{i} u_{j}\right)}{\partial x_{j}}+\alpha_{g} \frac{\partial p}{\partial x_{i}}=(1-\alpha) S_{g i}
\end{array}
$$

where the compatibility relation for volume fractions are, $\alpha_{l}+\alpha_{g}=1$ and volume fractions for the liquid and gas can therefore be expressed as $\alpha_{l}=\alpha$ and $\alpha_{g}=(1-\alpha)$. The source terms, $S_{l}$ and $S_{g}$, contain the hydro-static pressure terms as well as the second-order viscous terms. If Newtonian flow is assumed then the following holds

$$
\begin{array}{r}
S_{l}=\rho_{l} g_{i}+\mu_{l} \frac{\partial}{\partial x_{j}}\left(\frac{\partial u_{i}}{\partial x_{j}}\right) \\
S_{g}=\rho_{g} g_{i}+\mu_{g} \frac{\partial}{\partial x_{j}}\left(\frac{\partial u_{i}}{\partial x_{j}}\right)
\end{array}
$$
found

By adding Eq. (22) and Eq. (24) an averaged momentum equation for homogenous two-fluid flow is

$$
\frac{\partial\left(\rho u_{i}\right)}{\partial t}+\frac{\partial\left(\rho u_{i} u_{j}\right)}{\partial x_{j}}+\frac{\partial p}{\partial x}=S_{i}
$$

where the mean density and dynamic viscosity, expressed in terms of the volume fraction, are

$$
\begin{aligned}
\rho & =\alpha \rho_{l}+(1-\alpha) \rho_{g} \\
\mu & =\alpha \mu_{l}+(1-\alpha) \mu_{g}
\end{aligned}
$$

For the liquid-gas systems considered it is typically found that the acoustic velocities vary significantly (for example the values for air and water are respectively $343.2 \mathrm{~m} / \mathrm{s}$ and $1484 \mathrm{~m} / \mathrm{s}$ at $20^{\circ} \mathrm{C}$ ). As the compressibility of the fluid is a function of the square of the acoustic velocity, it is noted that degree to which the gas is compressible is much higher than the liquid. It would therefore be an acceptable assumption to treat the liquid as incompressible, $\rho_{l}=$ constant, and the gas as compressible. Dividing the liquid continuity equation, Eq. (21), by the constant $\rho_{l}$, the advection volume fraction equation is found

$$
\frac{\partial \alpha}{\partial t}+\frac{\partial\left(\alpha u_{j}\right)}{\partial x_{j}}=0
$$

Eq. (23), can be written as follows, when applying the product rule to the gas continuity equation and rearranging the terms,

$$
(1-\alpha) \frac{\partial \rho_{g}}{\partial t}+(1-\alpha) u_{j} \frac{\partial \rho_{g}}{\partial x_{j}}+\rho_{g} \frac{\partial u_{j}}{\partial x_{j}}-\rho_{g}\left[\frac{\partial \alpha}{\partial t}+\frac{\partial\left(\alpha u_{j}\right)}{\partial x_{j}}\right]=0
$$

It is noted that the last term in square brackets is the volume fraction equation and equal to zero. Considering the rate of acoustic wave propagation in the gas is much faster than the fluid velocity, an almost instantaneous 
pressure equalisation in the gas phase is realised. With the time scales considered, it is expected that within localised regions in the gas phase the pressure will only vary in time but not in space, $\rho_{g}=\rho_{g}(t)$. This is consistent with Song and Yuan, ${ }^{39}$ who presented a weakly compressible model for low Mach number, iso-thermal flow, where they showed via a non-dimensional analysis that the spatial derivative of pressure of the expanded continuity equation is negligibly small. The continuity equation for two fluid, immiscible flow is then

$$
\frac{(1-\alpha)}{\rho_{g}} \frac{\partial \rho_{g}}{\partial t}=-\frac{\partial u_{j}}{\partial x_{j}}
$$

and assuming iso-thermal flow the ideal gas law for weakly compressible flow is

$$
\rho-\rho_{o}=\frac{1}{c_{g}^{2}}\left(p-p_{o}\right)
$$

where $\rho_{o}$ and $p_{o}$ is the initial gas density and pressure.

\section{III.A.1. Numerical solution}

As the number of governing equations that need to be solved for the weakly compressible formulation is the same as for incompressible FSM, additional computational cost is kept to a minimum. The volume fraction equation remains unchanged from incompressible FSM and existing VOF free-surface capturing schemes can be employed. In the compressible formulation the continuity equation is however no longer linear, making it necessary to discretise the face flux using a third order upwind scheme with Sweby limiter ${ }^{40}$ to ensure an oscillatory free and stable solution.

A combination of the projected pressure (PP) method ${ }^{41}$ and the artificial compressibility (AC) method $^{42}$ as proposed by Nithiarasu ${ }^{43}$ is used to solve the equations in a matrix-free manner. The solver consist of three steps, first an intermediate momentum equation is solved viz.

$$
\frac{\Delta \rho u_{i}^{*}}{\Delta t}=-\left.\frac{\partial\left(\rho u_{i} u_{j}\right)}{\partial x_{j}}\right|^{n}+S_{i}^{n}
$$

where $\Delta \rho u_{i}^{*}=\rho u_{i}^{*}-\left.\rho u_{i}\right|^{n}$ and $\Delta t=t^{n+1}-t^{n}$.

There after the pressure is calculated using an implicit projected pressure equation with artificial compressibility

$$
\begin{aligned}
\frac{1}{c_{\tau}^{2}} \frac{p^{\tau+1}-p^{\tau}}{\Delta t_{\tau}}= & -\frac{\partial}{\partial x_{j}}\left[u_{j}^{\tau}+\Delta t\left(-u_{i} \frac{\partial u_{j}^{\tau}}{\partial x_{i}}-\frac{1}{\rho} \frac{\partial p^{\tau+1}}{\partial x_{j}}+\frac{S_{j}^{\tau}}{\rho}\right)\right] \\
& -\frac{(1-\alpha)}{\rho_{g}} \frac{1}{c_{g}^{2}} \frac{p^{\tau+1}-p^{n}}{\Delta t}
\end{aligned}
$$

where $c_{\tau}$ is the artificial acoustic velocity and $\Delta t_{\tau}$ the pseudo time step size.

Finally, the velocities are calculated from the corrected momentum equation which contain the updated values.

$$
\frac{\Delta \rho u_{i}^{n}}{\Delta t}=-\frac{\partial \rho u_{i} u_{j}}{\partial x_{j}}-\frac{\partial p^{n+1}}{\partial x_{i}}+S_{i}
$$

A Generalised Minimal Residual (GMRES) algorithm, with Lower-Upper Symmetric Gauss-Seidel (LUSGS) preconditioning is used to solve the projected pressure equation. ${ }^{44}$ It is found that the advance solver provides more or less a 100 times speedup in computational time from when a standard Jacobian type solver is used.

\section{III.B. Numerical evaluation}

In this section the newly derived weakly compressible FSM governing equations are validated against known analytical solutions. Furthermore, a comparative study is conducted to evaluate the difference in the numerical solution between incompressible FSM and a weakly compressible formulation. Unless otherwise 
Table 1: Material properties for the liquid and gas at $20^{\circ} \mathrm{C}$

\begin{tabular}{l|l|l} 
& Liquid (Water) & Gas (Air) \\
\hline Density $\left(\mathrm{kg} / \mathrm{m}^{3}\right)$ & 998 & 1.21 \\
Viscosity $(\mathrm{kg} /(\mathrm{ms}))$ & $1.002 \times 10^{-3}$ & $1.812 \times 10^{-6}$ \\
Acoustic velc $(\mathrm{m} / \mathrm{s})$ & $\infty$ & 343.2
\end{tabular}

stated, the material properties as given in Table 1 are used and gravitational acceleration is taken to be $g=9.81 \mathrm{~m} / \mathrm{s}^{2}$.

The first test case considered in the validation of the compressible formulation is the linear filling of a long two-dimensional rectangular tube. Water enters the tube at a constant velocity and compress an entrapped air pocket at the end of the tube as show in figure 9 . In the $5 \mathrm{~s}$ of the analysis, the initial water-air interface is propagated from $x=0.25 \mathrm{~m}$ to $x=0.75 \mathrm{~m}$. Three different uniform meshes are used: a coarse mesh with $3 \times 30$ nodes; a intermediate mesh with $5 \times 50$ nodes; and a fine mesh with $10 \times 100$ nodes. Slip boundary conditions are specified on the sides of the tube.

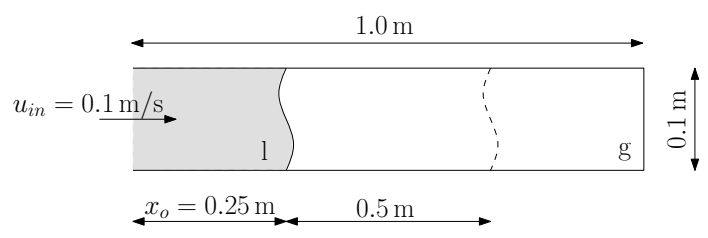

Figure 9: Schematic of the linear filled tube

In figure 10a the averaged pressure in the compressed air pocket for the three different meshes are plotted as a function of time. The figure shows the exponential growth in pressure over time and that as the mesh is refined the numerical result approaches the analytical solution. In figure 10b the discontinuous velocity profile, for the coarse mesh, is compared to the analytical solution at various time frames during the analysis. From this it is noted that an oscillatory free solution is obtained, where the velocity is constant within the incompressible fluid and reduces linearly in the compressible gas.

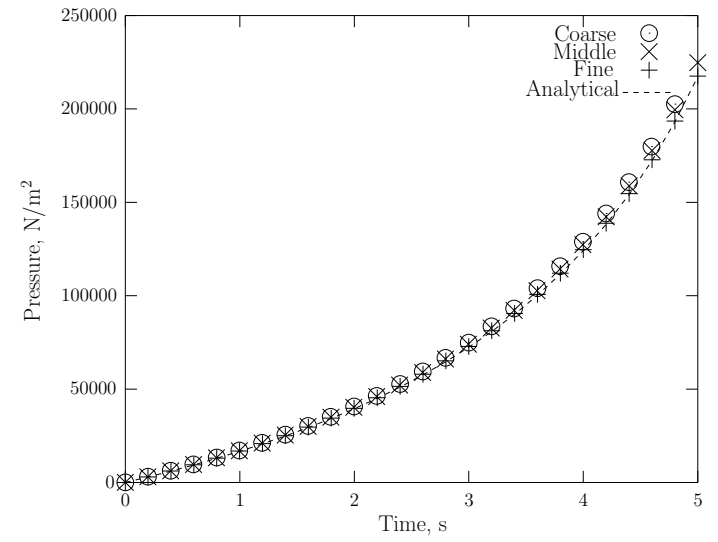

(a) Non-linear increase of pressure inside the tube as a(b) Velocity profile at $t=1 \mathrm{~s}, t=2 \mathrm{~s}, t=3 \mathrm{~s}$ and $t=4 \mathrm{~s}$ function of time

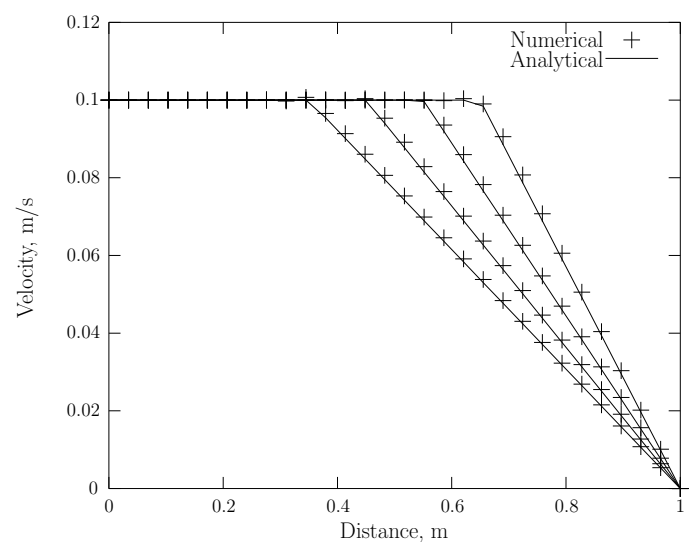

(numerical result on the coarse mesh)

Figure 10: Comparison of the numerical and analytical results for the linear filling of a rectangular 2D tube

Having validated the developed technology, the next test-case involves an application study. For this purpose a water pocket trapped between two air pockets in a long horizontal tube under variable sideways excitation, as shown in figure 11, is modelled. The water pocket is initially at the centre of the tube. Two different horizontal accelerations are applied, of which the first involves a smooth sinusoidal acceleration, 
and the second, a step function which is sharply ramped up and down. The accelerations can be written as

$$
\begin{aligned}
& a_{x}^{\text {sin }}=10 \sin (2 \pi t) \\
& a_{x}^{\text {step }}= \begin{cases}10 & \text { if } t>0.1 \text { or } t<0.5 \\
0 & \text { if } t<0.1 \text { or } t>0.5\end{cases}
\end{aligned}
$$

where $a_{x}$ is the lateral acceleration and $t$ is time in seconds.

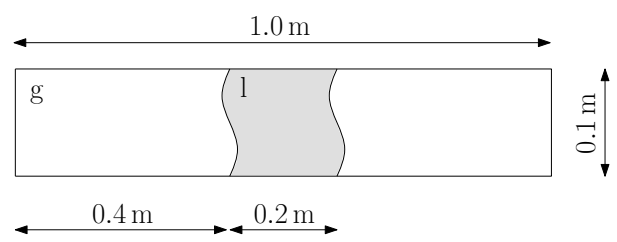

Figure 11: Schematic of the entrapped pocket of water under horizontal excitation

In figure $12 \mathrm{a}$ and $12 \mathrm{~b}$, plots of the left side wall pressures as a function of time are shown, where numerical results for different acoustic velocities is compared to an incompressible hydro-static pressure model, which assumes the pressure on the side wall is the product of the mass times the acceleration.

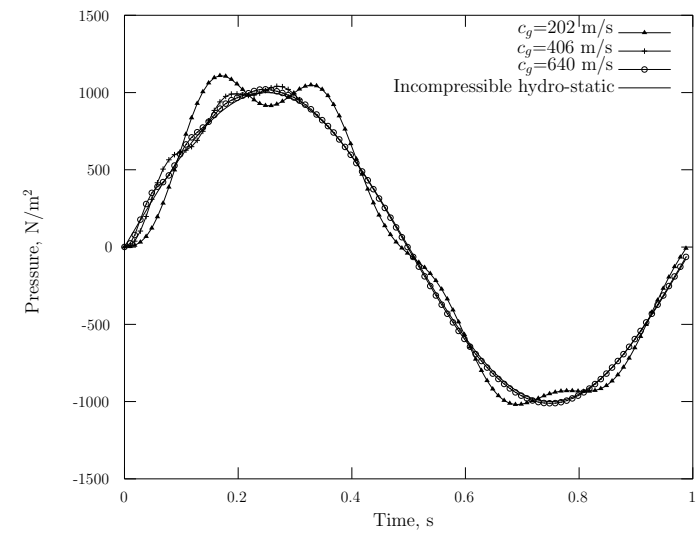

(a) Sinusoidal acceleration

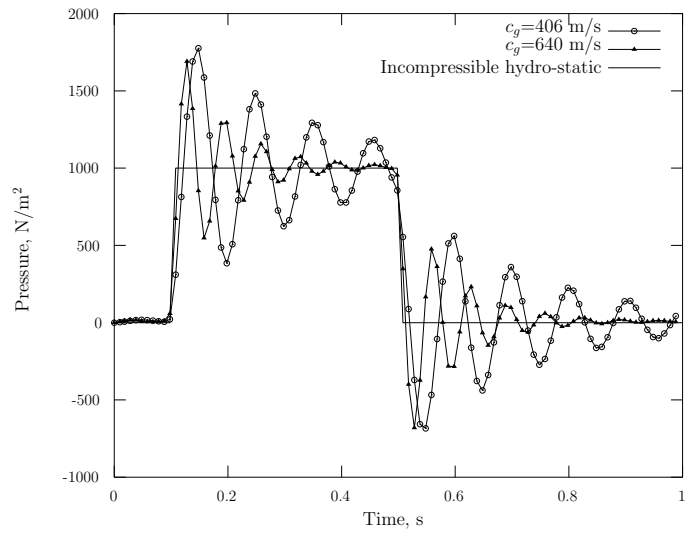

(b) Stepwise acceleration

Figure 12: Sidewall pressure measured as a function of time, for the entrapped pocket of water under horizontal excitation

From the figures it is noted that the numerical solution approach the incompressible hydro-static solution as the gas acoustic velocity increases. Similar to a mass spring system, the gas absorbs the energy initially, resulting in a lag in the pressure measured on the side-wall. However, the pressure then tends to over shoot the predicted incompressible flow hydro-static pressures. In the case of the step function, the maximum pressure measured for compressible flow is almost twice as high as the incompressible hydro-static pressure. Furthermore, the frequency of the pressure oscillations increases along with the acoustic velocity.

Finally the new weakly compressible formulation is evaluated by means of a comparative study, where a partially filled tank with a baffle configuration, as shown in figure 13, under lateral excitation is considered. The tank is $70 \%$ filled with liquid and is subjected to lateral sinusiodal excitation with an amplitude of 8 $\mathrm{m} / \mathrm{s}^{2}$ and a frequency of $2 \mathrm{~Hz}$. For the analysis a 5000 node hybrid structured mesh is used.

The compressible and incompressible formulations are compared by evaluating the average side wall pressures calculated. As only the change in pressure can be computed for the incompressible flow and not the absolute pressures, the difference in average left and right side wall pressures is plotted in figure 14 and difference in the baffle side wall pressures is plotted in figure 15. The most notable difference between the compressible and incompressible formulations is the sharp pressure spikes at $t=0.5 \mathrm{~s}$ and $t=0.62 \mathrm{~s}$, which corresponds to the times that liquid suddenly covers the top baffle opening. As the incompressible 


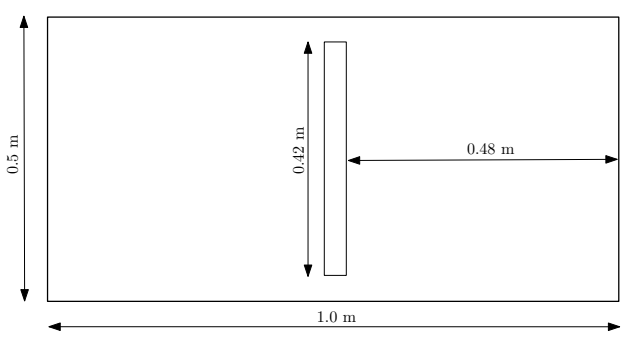

(a) Schematic

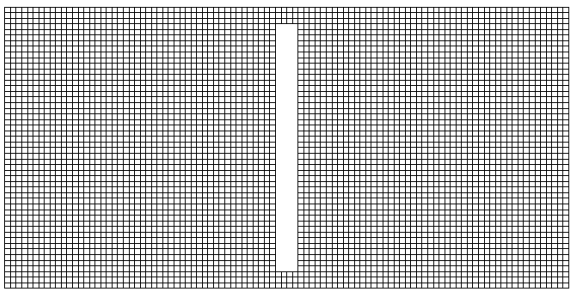

(b) Mesh

Figure 13: PaSchemLatTankBafflek with baffle subjected to sinusiodal lateral excitation

formulation can not account for the compression of the gas it results in sharp spikes in the pressure. For the weakly compressible formulation the maximum pressure calculated at these points are slightly less, but as is to be expected, a smooth pressure oscillation is noted as the gas stores and releases energy. This results in larger variations in the average forces translated to the baffle and tank side walls. For the weakly compressible formulation it is noted that there is a slight improvement in the computational time. As the latter takes into account the compressibility of the gas, it is expected that the scheme softens the numerical system leading to a quicker convergence of the solution.

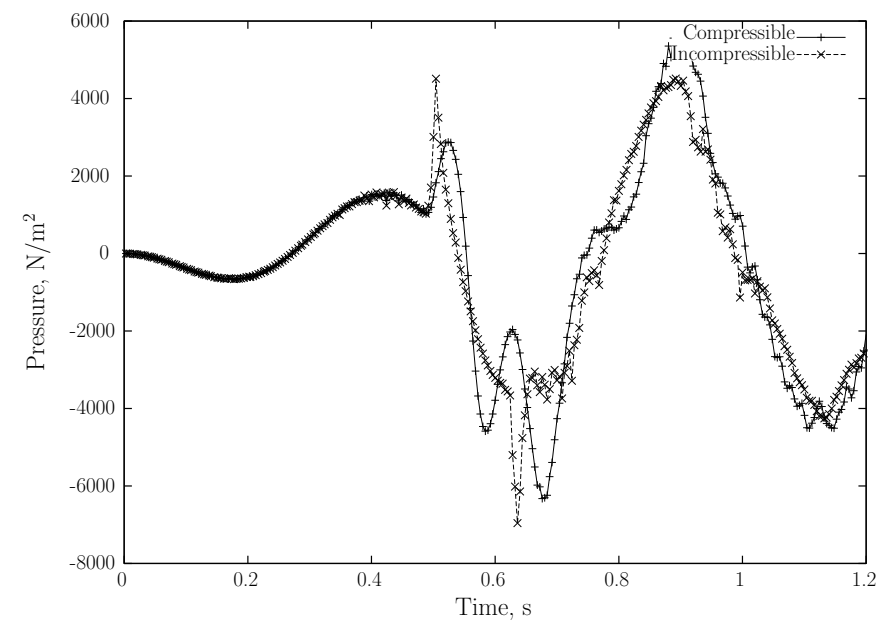

Figure 14: Difference in average pressure between the left and right side wall of the partially filled tank subjected to lateral excitation

\section{Conclusion}

The aim of this work was to extend the capabilities of existing VOF FSM schemes in two ways. The first involved improving the free-surface interface capturing scheme and the second looked at accounting for the compressible properties of a gas by means of a weakly compressible formulation. A new fast compressive surface capturing formulation is presented that proved to ensure that a sharp interface is maintained at higher Courant numbers while it prevents the interface from wrinkling. It is shown that FCSCF provides a significant increased accuracy in standard bench-marked test cases such as the rotating key, but also in violent flow condition when coupled to a incompressible flow solver. The weakly compressible VOF formulation is validated and is shown to be accurate. Furthermore, despite the large numerical discontinuities, a oscillatory free solution is achieved and the solver proved to be stable and robust.An addition, when considering a $2 \mathrm{D}$ sloshing problem the weakly compressible formulation is found to be faster to solve than the incompressible case. 


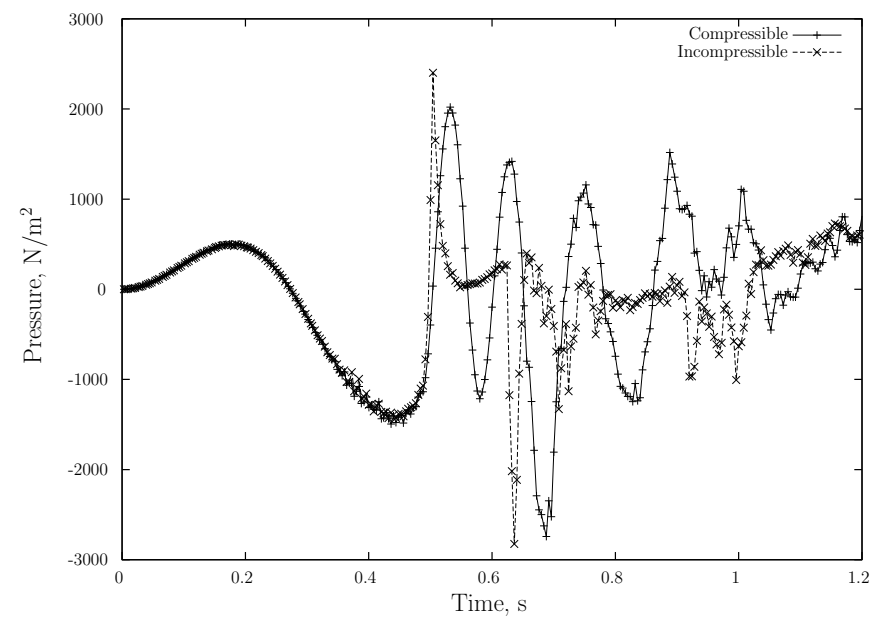

Figure 15: Difference in average pressure between the left and right baffle walls of the partially filled tank subjected to lateral excitation

\section{References}

${ }^{1}$ Terashima, H. and Tryggvason, G., "A front-tracking method with projected interface conditions for compressible multifluid flows," Computers \& Fluids, Vol. 39, No. 10, 2010, pp. $1804-1814$.

${ }^{2}$ Chen, Y., Price, W., and Temarel, P., "Numerical simulation of liquid sloshing in LNG tanks using a compressible two-fluid flow model," Proceedings of the Nineteenth International Offshore and Polar Engineering Conference, 2009.

${ }^{3}$ Darwish, M. and Moukalled, F., "Convective schemes for capturing interfaces of free-surface flows on unstructured grids," Numerical Heat Transfer, Part B: Fundamentals, Vol. 49, No. 1, 2006, pp. 19-42.

${ }^{4}$ de Sousa, F. S., Mangiavacchi, N., Nonato, L. G., Castelo, A., Tom, M. F., Ferreira, V. G., Cuminato, J. A., and McKee, S., "A front-tracking/front-capturing method for the simulation of 3D multi-fluid flows with free surfaces," Journal of Computational Physics, Vol. 198, No. 2, 2004, pp. 469 - 499.

${ }^{5}$ Hirt, C. and Nichols, B., "Volume of fluid (VOF) method for the dynamics of free boundaries*1," Journal of computational physics, Vol. 39, No. 1, 1981, pp. 201-225.

${ }^{6}$ Ubbink, O. and Issa, R. I., "A Method for Capturing Sharp Fluid Interfaces on Arbitrary Meshes," Journal of Computational Physics, Vol. 153, 1999, pp. 26-50.

${ }^{7}$ Malan, A. G., Meyer, J. P., and Lewis, R. W., "Modelling Non-Linear Heat Conduction via a Fast Matrix-Free Implicit Unstructured-Hybrid Algorithm," Computer Methods in Applied Mechanics and Engineering, Vol. 196, No. 45-48, 2007, pp. $4495-4504$.

${ }^{8}$ Zhao, Y. and Zhang, B., "A High-Order Characteristics Upwind FV Method for Incompressible Flow and Heat Transfer Simulation on Unstructured Grids," International Journal of Numerical Methods in Engineering, Vol. 37, 1994, pp. 3323-3341.

${ }^{9}$ Ferziger, J. H. and Peric, M., Computational Methods for Fluid Dynamics, Springer-Verlag, New York, 1999.

${ }^{10}$ Clarke, A. and Issa, R. I., "A numerical model of slug flow in vertical tubes," Computers and Fluids, Vol. 26, No. 4, 1997, pp. $395-415$.

${ }^{11}$ Popinet, S. and Zaleski, S., "A front-tracking algorithm for accurate representation of surface tension," International Journal for Numerical Methods in Fluids, Vol. 30, No. 6, 1999, pp. 775-793.

${ }^{12}$ Unverdi, S. O. and Tryggvason, G., "A front-tracking method for viscous, incompressible, multi-fluid flows," Journal of Computational Physics, Vol. 99, No. 1, 1992, pp. 180 - 180.

${ }^{13}$ López, J. and Hernández, J., "On reducing interface curvature computation errors in the height function technique," Journal of Computational Physics, Vol. 229, No. 13, 2010, pp. 4855 - 4868.

${ }^{14}$ Farmer, J., Martinelli, L., and Jameson, A., "Fast multigrid method for solving incompressible hydrodynamic problems with free surfaces," AIAA journal, Vol. 32, No. 6, 1994, pp. 1175-1182.

${ }^{15}$ Sethian, J., "Level set methods and fast marching methods," Journal of Computing and Information Technology, Vol. 11, No. 1, 2003, pp. 1-2.

${ }^{16}$ Sussman, M., Almgren, A., Bell, J., Colella, P., Howell, L., and Welcome, M., "An Adaptive Level Set Approach for Incompressible Two-Phase Flows* 1," Journal of Computational Physics, Vol. 148, No. 1, 1999, pp. 81-124.

${ }^{17}$ Raessi, M., Mostaghimi, J., and Bussmann, M., "A volume-of-fluid interfacial flow solver with advected normals," Computers 8 Fluids, Vol. 39, No. 8, 2010, pp. $1401-1410$.

${ }^{18}$ Gopala, V. R. and van Wachem, B. G., "Volume of fluid methods for immiscible-fluid and free-surface flows," Chemical Engineering Journal, Vol. 141, No. 1-3, 2008, pp. 204-221.

${ }^{19}$ Cummins, S. J., Francois, M. M., and Kothe, D. B., "Estimating curvature from volume fractions," Computers 83 Structures, Vol. 83, No. 6-7, 2005, pp. $425-434$.

${ }^{20}$ Muzaferija, S., Peric, M., Sames, P., and Schellin, T., "A two-fluid Navier-Stokes solver to simulate water entry," Proc 22nd Symposium on Naval Hydrodynamics, 1998, pp. 277-289. 
${ }^{21}$ Rusche, H., Computational fluid dynamics of dispersed two-phase flows at high phase fractions, Ph.D. thesis, Imperial College, University of London, 2002.

${ }^{22}$ Jasak, H. and Weller, H., "Interface tracking capabilities of the inter-gamma differencing scheme," Tech. rep., Technical report, CFD research group, Imperial College, London, 1995, 1995.

${ }^{23}$ Leonard, B. P., "The ULTIMATE conservative difference scheme applied to unsteady one-dimensional advection," Computer Methods in Applied Mechanics and Engineering, Vol. 88, No. 1, 1991, pp. $17-74$.

${ }^{24}$ Ubbink, O., Numerical prediction of two fluid systems with sharp interfaces, Ph.D. thesis, Department of Mechanical engineering, Imperial College, London, 1997.

${ }^{25}$ Tsui, Y.-Y., Lin, S.-W., Cheng, T.-T., and Wu, T.-C., "Flux-blending schemes for interface capture in two-fluid flows," International Journal of Heat and Mass Transfer, Vol. 52, No. 23-24, 2009, pp. $5547-5556$.

${ }^{26}$ Wacławczyk, T. and Koronowiczy, T., "Comparison of CICSAM and HRIC High-Resolution Schemes for Interface Capturing," Journal of Theoretical and Applied Mechanics, Vol. 46, No. 2, 2008, pp. 325-345.

${ }^{27}$ Cassidy, D. A., Edwards, J. R., and Tian, M., "An investigation of interface-sharpening schemes for multi-phase mixture flows," Journal of Computational Physics, Vol. 228, No. 16, 2009, pp. 5628 - 5649.

${ }^{28}$ Wacławczyk, T. and Koronowiczy, T., "Modeling of the Wave Breaking With CICSAM and HRIC High-Resolution Schemes," European Conference on Computational Fluid Dynamics ECCOMAS CFD, edited by S. Wesseling, E. Oñate, and J. Périaux, 2006.

${ }^{29}$ Zalesak, S., "Fully multidimensional flux-corrected transport algorithms for fluids," Journal of Computational Physics, Vol. 31, No. 3, 1979, pp. 335-362.

${ }^{30}$ Godderidge, B., Turnock, S., Earl, C., and Tan, M., "The effect of fluid compressibility on the simulation of sloshing impacts," Ocean Engineering, Vol. 36, No. 8, 2009, pp. 578-587.

${ }^{31}$ Wemmenhove, R., Luppes, R., Veldman, A., and Bunnik, T., "Numerical simulation of sloshing in LNG tanks with a compressible two-phase model," 26th International Conference on Offshore Mechanics and Arctic Engineering, San Diego, USA, 2007.

${ }^{32}$ Faltinsen, O., Rognebakke, O., and Timokha, A., "Classification of three-dimensional nonlinear sloshing in a square-base tank with finite depth," Journal of Fluids and Structures, Vol. 20, 2005, pp. 81-103.

${ }^{33}$ Lugni, C., Brocchini, M., and Faltinsen, O., "Wave impact loads: The role of the flip-through," Physics of fluids, Vol. 18, 2006.

${ }^{34}$ Bullock, G., Obhrai, C., Peregrine, D., and Bredmose, H., "Violent breaking wave impacts. Part 1: Results from largescale regular wave tests on vertical and sloping walls," Coastal Engineering, Vol. 54, No. 8, 2007, pp. 602-617.

${ }^{35}$ Liou, M., Nguyen, L., Chang, C., and Theofanous, T., "How to solve compressible multifluid equations: a simple, robust and accurate method," AIAA paper, Vol. 4456, 2007, pp. 2007.

${ }^{36}$ Murrone, A. and Guillard, H., "A five equation reduced model for compressible two phase flow problems," Journal of Computational Physics, Vol. 202, No. 2, 2005, pp. 664-698.

${ }^{37}$ Romenski, E. and Toro, E., "Compressible two-phase flows: two-pressure models and numerical methods," Comput. Fluid Dyn. J, Vol. 13, 2004, pp. 403-416.

${ }^{38}$ Dias, F., Dutykh, D., and Ghidaglia, J., "A two-fluid model for violent aerated flows," Computers $8 \mathcal{G}$ fluids, Vol. 39, No. 2, 2010, pp. 283-293.

${ }^{39}$ Song, C. and Yuan, M., "A weakly compressible flow model and rapid convergence methods," Journal of Fluids Engineering, Vol. 110, No. 4, 1988, pp. 441-455.

${ }^{40}$ Waterson, N. and Deconinck, H., "Design principles for bounded higher-order convection schemes-a unified approach," Journal of Computational Physics, Vol. 224, No. 1, 2007, pp. 182-207.

${ }^{41}$ Patankar, S. V., Numerical Heat Transfer and Fluid Flow, McGraw-Hill, New York, 1980.

${ }^{42}$ Chorin, A. J., "A Numerical Method for Solving Incompressible Viscous Flow Problems," Journal of Computational Physics, Vol. 2, 1967, pp. 12-26.

${ }^{43}$ Nithiarasu, P., "An efficient artificial compressibility (AC) scheme based on the characteristic based split (CBS) method for incompressible flow," International Journal for Numerical Methods in Engineering, Vol. 56, No. 13, 2003, pp. $1815-1845$.

${ }^{44}$ Malan, A. and Oxtoby, O., "A Parallel Free-Surface-Modelling Technology for Application to Aircraft Fuel-Sloshing," Lisbon, ECCOMAS CFD-Fifth European Conference on Computational Fluid Dynamics, Lisbon, Vol. 14. 